\title{
Stability of a Nonlinear Stochastic Epidemic Model with Transfer from Infectious to Susceptible
}

\author{
Yanmei Wang $\mathbb{D}^{1,2}$ and Guirong Liu $\mathbb{D}^{1}$ \\ ${ }^{1}$ School of Mathematical Sciences, Shanxi University, Taiyuan, Shanxi 030006, China \\ ${ }^{2}$ School of Applied Mathematics, Shanxi University of Finance and Economics, Taiyuan, Shanxi 030006, China
}

Correspondence should be addressed to Guirong Liu; lgr5791@sxu.edu.cn

Received 3 April 2020; Revised 16 May 2020; Accepted 19 May 2020; Published 31 May 2020

Guest Editor: Carlos Arturo Loredo Villalobos

Copyright (C) 2020 Yanmei Wang and Guirong Liu. This is an open access article distributed under the Creative Commons Attribution License, which permits unrestricted use, distribution, and reproduction in any medium, provided the original work is properly cited.

We investigate a stochastic SIRS model with transfer from infectious to susceptible and nonlinear incidence rate. First, using stochastic stability theory, we discuss stochastic asymptotic stability of disease-free equilibrium of this model. Moreover, if the transfer rate from infectious to susceptible is sufficiently large, disease goes extinct. Then, we obtain almost surely exponential stability of disease-free equilibrium, which implies that noises can lead to extinction of disease. By the Lyapunov method, we give conditions to ensure that the solution of this model fluctuates around endemic equilibrium of the corresponding deterministic model in average time. Furthermore, numerical simulations show that the fluctuation increases with increase in noise intensity. Finally, these theoretical results are verified by numerical simulations. Hence, noises play a vital role in epidemic transmission. Our results improve and extend previous related results.

\section{Introduction}

Mathematical models have become a crucial tool in understanding dynamics of population growth [1-3]. In recent decades, some realistic mathematical models have been established to investigate dynamics of epidemic [4-10]. In order to simulate epidemic transmission process, many dynamic models have been established, such as SIS, SEIR, and SIRS models [11-13]. In these models, the incidence rate is crucial. Classical disease transmission models adopt the standard or bilinear incidence rate. However, in the course of epidemic propagation, nonlinear incidence may be more realistic than other incidence rates [14]. In addition, infected individuals may recover after a period of treatment or become susceptible individuals directly due to transient antibody. In [15], a deterministic SIRS model with transfer from infectious to susceptible and nonlinear incidence can be modeled as follows:

$$
\left\{\begin{array}{l}
\dot{S}=\Lambda+\gamma_{1} I+\delta R-\beta S f(I)-\mu S, \\
\dot{I}=\beta S f(I)-\left(\alpha+\gamma_{1}+\gamma_{2}+\mu\right) I, \\
\dot{R}=\gamma_{2} I-(\delta+\mu) R .
\end{array}\right.
$$

Here, $S, I$, and $R$ denote numbers of susceptible, infectious, and recovered individuals, respectively. $\Lambda$ is the recruitment rate of susceptible; $\beta$ denotes the disease propagation coefficient; $\mu$ and $\alpha$ denote, respectively, the natural death rate and mortality caused by the disease; $\delta$ denotes the immunity loss rate; $\gamma_{1}$ represents the transfer rate from infectious to susceptible; $\gamma_{2}$ denotes the recovery rate of infectious individuals. In addition, $\Lambda>0, \mu>0, \gamma_{1} \geq 0$, $\gamma_{2} \geq 0, \delta \geq 0$, and $\alpha \geq 0$.

From [15], (1) has disease-free equilibrium $E_{0}(\Lambda / \mu, 0,0)$ which is globally asymptotic stable in $\left\{(S, I, R) \in \mathbb{R}_{+}^{3}: S+\right.$ $I+R \leq \Lambda / \mu\} \quad$ if $\quad \mathscr{R}_{0}=(\beta \Lambda) /\left[\mu\left(\gamma_{1}+\gamma_{2}+\mu+\alpha\right)\right]<1$. If $\mathscr{R}_{0}>1$, there exists a globally asymptotic stable endemic equilibrium $E^{*}\left(S^{*}, I^{*}, R^{*}\right)$.

However, dynamics of epidemic is often disturbed by some random factors. Hence, stochastic epidemic models are more realistic and have attracted much attention [16-19]. In [20], the authors discussed threshold behavior for a stochastic SIS model. In [21], asymptotic properties of a stochastic SIR model were considered. In [22, 23], the authors investigated persistence and extinction for a stochastic SIRS model. In [24], the authors studied stability of a stochastic SIRS model. Fatini et al. [25] considered stochastic 
stability and instability for a stochastic SIR model. Recently, Wang et al. [26] established a stochastic SIRS epidemic model:

$$
\left\{\begin{array}{l}
\mathrm{d} S=\left[\Lambda+\gamma_{1} I+\delta R-\beta S f(I)-\mu S\right] \mathrm{d} t-\sigma S f(I) \mathrm{d} B(t), \\
\mathrm{d} I=\left[\beta S f(I)-\left(\alpha+\gamma_{1}+\gamma_{2}+\mu\right) I\right] \mathrm{d} t+\sigma S f(I) \mathrm{d} B(t), \\
\mathrm{d} R=\left[\gamma_{2} I-(\mu+\delta) R\right] \mathrm{d} t
\end{array}\right.
$$

with initial values $S_{0}>0, I_{0}>0$, and $R_{0}>0$. Here, $B(t)$ represents Brownian motion on $(\Omega, \mathscr{F}, \mathbb{P})$ which is a complete probability space. $\sigma^{2}$ denotes the intensity of $B(t)$. Other parameters are defined as (1). Model (2) covers many stochastic models as particular cases (see, for example, $[15,22,27])$. In [26], extinction and persistence are obtained.

As is well known, stability of the dynamic system means that solutions are insensitive to small changes of initial value. Hence, stability is one of the important topics encountered in applications. However, because of the complexity of stochastic dynamics, there are not many results on stability of stochastic differential equations.

Motivated by the above work, we consider (2) and obtain stochastic stability of disease-free equilibrium and asymptotic behavior around endemic equilibrium of corresponding deterministic model (1).

Throughout this paper, we give the following hypotheses:

$\left(\mathbf{H}_{1}\right) f$ is locally Lipschitz on $[0, \infty) ; f(I)>0$ for $I>0$; $f(0)=0$

$\left(\mathbf{H}_{2}\right) \lim _{I \longrightarrow 0^{+}} f(I) / I=1$ and $f(I) / I$ is nonincreasing on $(0, \infty)$

From $\left(\mathbf{H}_{1}\right), \quad(2)$ has disease-free equilibrium $E_{0}(\Lambda / \mu, 0,0)$. By $\left(\mathbf{H}_{2}\right)$, if $I \in(0, \infty)$, then

$$
I \geq f(I) \text {. }
$$

\section{Preliminaries}

We will give some definitions and lemmas. Consider

$$
\mathrm{d} x=F(x, t) \mathrm{d} t+G(x, t) \mathrm{d} W(t), t \geq t_{0} .
$$

Here, $F$ and $G$ are, respectively, $\mathbb{R}^{d}$-valued and $\mathbb{R}^{d \times n}$-valued functions defined on $\mathbb{R}^{d} \times\left[t_{0}, \infty\right)$ and $\mathbb{R}^{d} \times\left[t_{0}, \infty\right) . \quad\{W(t)\}$ denotes $n$-dimensional Brownian motion on $\left(\Omega, \mathscr{F},\left\{\mathscr{F}_{t}\right\}_{t \geq 0}, \mathbb{P}\right)$. Assume that existence-anduniqueness theorem is fulfilled. For $t \geq t_{0}, G(0, t)=0$ and $F(0, t)=0$. Denote $\overline{\mathbb{R}}_{+}=\mathbb{R}_{+} \cup\{0\}$ and $\mathrm{K}=\left\{\mu \in C\left(\overline{\mathbb{R}}_{+}, \overline{\mathbb{R}}_{+}\right)\right.$ $: \mu$ is nondecreasing; $\mu(0)=0 ; \mu(r)>0$ if $r>0\}$. Set $S_{h}=\left\{x \in \mathbb{R}^{d}:|x|<h\right\}$.

\section{Definition 1 ([[28], p.108])}

(i) Assume that $V$ is continuous on $S_{h} \times\left[t_{0}, \infty\right)$ and $V(0, t) \equiv 0$. If there is $\mu \in \mathrm{K}$ such that, for $(x, t) \in S_{h} \times\left[t_{0}, \infty\right)$,

$$
V(x, t) \geq \mu(|x|),
$$

then $V$ is positive-definite. In addition, $V$ is negativedefinite if $-V$ is positive-definite.

(ii) Assume that $V$ is nonnegative and continuous on $S_{h} \times\left[t_{0}, \infty\right)$. If there is $\mu \in \mathrm{K}$, satisfying for $(x, t) \in S_{h} \times\left[t_{0}, \infty\right)$,

$$
V(x, t) \leq \mu(|x|),
$$

then $V$ is decrescent.

Definition 2 ([[28], p.110])

(i) If for any $r>0$ and $\varepsilon \in(0,1)$, there is $\delta=\delta\left(\varepsilon, r, t_{0}\right)>0$, satisfying for any $x_{0} \in \mathbb{R}^{d}$ with $\left|x_{0}\right|<\delta$,

$$
\mathbb{P}\left\{\left|x\left(t ; t_{0}, x_{0}\right)\right|<r \text { for } t \geq t_{0}\right\} \geq 1-\varepsilon,
$$

then trivial solution to (4) is stochastically stable.

(ii) The trivial solution to (4) is stochastically asymptotically stable if it is stochastically stable, and for any $\varepsilon \in(0,1)$, there is $\delta_{0}=\delta_{0}\left(\varepsilon, t_{0}\right)>0$ satisfying

$$
\mathbb{P}\left\{\lim _{t \longrightarrow \infty} x\left(t ; t_{0}, x_{0}\right)=0\right\} \geq 1-\varepsilon,
$$

whenever $\left|x_{0}\right|<\delta_{0}$.

(iii) If for any $x_{0} \in G \subset \mathbb{R}^{d}$, $\lim \sup _{t \rightarrow \infty}\left(\ln \left|x\left(t ; t_{0}, x_{0}\right)\right|\right) / t<0$ a.s., then trivial solution to (4) is almost surely exponentially stable in $G$.

Lemma 1 ([[28], p.112]). If $V \in C^{2,1}\left(S_{h} \times\left[t_{0}, \infty\right) ; \overline{\mathbb{R}}_{+}\right)$is positive-definite and decrescent, and $L V(x, t)$ is negativedefinite, then trivial solution to (4) is stochastically asymptotically stable. holds.

By Theorem 1 and Remark 1 in [26], the following result

Lemma 2 (see [26]). For $\left(S_{0}, I_{0}, R_{0}\right) \in \mathbb{R}_{+}^{3}$, there is a unique global positive solution to (2). Moreover,

$$
D=\left\{(S, I, R) \in \mathbb{R}_{+}^{3}: S+I+R \leq \frac{\Lambda}{\mu}\right\}
$$

is positively invariant.

\section{Stability of Disease-Free Equilibrium}

In epidemiology, stability has important practical significance.

Theorem 1. If $\mathscr{R}_{0}<1, \quad\left(\sigma^{2} \Lambda^{2}\right) /\left(2 \mu^{2}\right)<\left(\mu+\gamma_{1}+\gamma_{2}+\alpha\right)$ $\left(1-\mathscr{R}_{0}\right)$, then disease-free equilibrium $E_{0}$ to (2) is stochastically asymptotically stable in D.

Proof. Denote $x=\left(x_{1}, x_{2}, x_{3}\right)=(-S+\Lambda / \mu, I, R)$. Define Lyapunov function 


$$
V_{2}(x)=x_{1}^{2}+b x_{2}^{2}+x_{3}^{2}
$$

for $(S, I, R) \in D$, where $b>0$ is to be chosen later. Clearly, $V_{2}$ is positive-definite. Note that $V_{2}(x) \leq(1 \vee b)|x|^{2}=: \mu(|x|)$. From Definition 1 (ii), it follows that $V_{2}$ is decrescent. Now, we show that $L V_{2}$ is negative-definite.

From Itô formula, for any $(S, I, R) \in D$,

$$
\begin{aligned}
\mathrm{LV}_{2}(x)= & -2\left(\frac{\Lambda}{\mu}-S\right)\left(\Lambda-\mu S-\beta S f(I)+\delta R+\gamma_{1} I\right) \\
& +2 b I\left[\beta S f(I)-\left(\mu+\alpha+\gamma_{1}+\gamma_{2}\right) I\right] \\
& +2 R\left[\gamma_{2} I-(\delta+\mu) R\right]+(1+b)(\sigma S f(I))^{2} \\
\leq & -2 \mu\left(\frac{\Lambda}{\mu}-S\right)^{2}+2\left(\frac{\Lambda}{\mu}-S\right)\left(\beta S-\gamma_{1}\right) I \\
& +\left[2 b \beta S-2 b\left(\mu+\gamma_{1}+\gamma_{2}+\alpha\right)+(1+b) \sigma^{2} S^{2}\right] I^{2} \\
& +2 \gamma_{2} I R-2 \delta\left(\frac{\Lambda}{\mu}-S\right) R-2(\delta+\mu) R^{2} \\
\leq & -2 \mu\left(\frac{\Lambda}{\mu}-S\right)^{2}+2\left(\frac{\Lambda}{\mu}-S\right)\left(\frac{\beta \Lambda}{\mu}-\gamma_{1}\right) I \\
& +\left[\frac{2 b \beta \Lambda}{\mu}-2 b\left(\mu+\gamma_{1}+\gamma_{2}+\alpha\right)+\frac{(1+b) \sigma^{2} \Lambda^{2}}{\mu^{2}}\right] I^{2} \\
& +2 \gamma_{2} I R-2 \delta(I+R) R-2(\delta+\mu) R^{2} .
\end{aligned}
$$

Obviously, we have

$$
\begin{gathered}
-\mu\left(\frac{\Lambda}{\mu}-S\right)^{2}+\left(\frac{\Lambda}{\mu}-S\right)\left(\frac{\beta \Lambda}{\mu}-\gamma_{1}\right) I \\
=-\mu\left[\left(\frac{\Lambda}{\mu}-S\right)-\frac{1}{2 \mu^{2}}\left(\beta \Lambda-\gamma_{1} \mu\right) I\right]^{2}+\frac{1}{4 \mu^{3}}\left(\beta \Lambda-\gamma_{1} \mu\right)^{2} I^{2} \\
-(\mu+2 \delta) R^{2}+\left(\gamma_{2}-\delta\right) I R=-(\mu+2 \delta)\left[R-\frac{\gamma_{2}-\delta}{2(\mu+2 \delta)} I\right]^{2} \\
+\frac{\left(\gamma_{2}-\delta\right)^{2}}{4(\mu+2 \delta)} I^{2} .
\end{gathered}
$$

Substituting (12) and (13) into (11) yields

$$
\begin{aligned}
L V_{2}(x) \leq & -2 \mu\left[\left(\frac{\Lambda}{\mu}-S\right)-\frac{1}{2 \mu^{2}}\left(\beta \Lambda-\gamma_{1} \mu\right) I\right]^{2} \\
& +\frac{1}{2 \mu^{3}}\left(\beta \Lambda-\gamma_{1} \mu\right)^{2} I^{2}+\frac{\left(\gamma_{2}-\delta\right)^{2}}{2(\mu+2 \delta)} I^{2}+\frac{\sigma^{2} \Lambda^{2}}{\mu^{2}} I^{2} \\
& -2 b\left[\mu+\gamma_{1}+\gamma_{2}+\alpha-\frac{\beta \Lambda}{\mu}-\frac{\sigma^{2} \Lambda^{2}}{2 \mu^{2}}\right] I^{2} \\
& -2(\mu+2 \delta)\left[R-\frac{\gamma_{2}-\delta}{2(\mu+2 \delta)} I\right]^{2} \leq \\
& -2 \mu\left[\left(\frac{\Lambda}{\mu}-S\right)-\frac{1}{2 \mu^{2}}\left(\beta \Lambda-\gamma_{1} \mu\right) I\right]^{2} \\
& -2(\mu+2 \delta)\left[R-\frac{\gamma_{2}-\delta}{2(\mu+2 \delta)} I\right]^{2} \\
& -\left[2 b\left(\mu+\gamma_{1}+\gamma_{2}+\alpha-\frac{\beta \Lambda}{\mu}-\frac{\sigma^{2} \Lambda^{2}}{2 \mu^{2}}\right)\right. \\
& \left.-\frac{1}{2 \mu^{3}}\left(\beta \Lambda-\gamma_{1} \mu\right)^{2}-\frac{\sigma^{2} \Lambda^{2}}{\mu^{2}}-\frac{\left(\gamma_{2}-\delta\right)^{2}}{2(\mu+2 \delta)}\right] I^{2} .
\end{aligned}
$$

Note $\left(\sigma^{2} \Lambda^{2}\right) /\left(2 \mu^{2}\right)<\left(\alpha+\gamma_{1}+\gamma_{2}+\mu\right)\left(1-\mathscr{R}_{0}\right)$. Then, $\alpha+\gamma_{1}+\gamma_{2}+\mu-(\beta \Lambda) / \mu-\left(\sigma^{2} \Lambda^{2}\right) /\left(2 \mu^{2}\right)>0$. Take

$$
b>\frac{\left(1 / 2 \mu^{3}\right)\left(\beta \Lambda-\gamma_{1} \mu\right)^{2}+\sigma^{2} \Lambda^{2} / \mu^{2}+\left(\gamma_{2}-\delta\right)^{2} / 2(\mu+2 \delta)}{2\left(\mu+\gamma_{1}+\gamma_{2}+\alpha-(\beta \Lambda / \mu)-\left(\sigma^{2} \Lambda^{2} / 2 \mu^{2}\right)\right)} .
$$

This yields that $L V_{2}$ is negative-definite. From Lemma 1, $E_{0}$ is stochastically asymptotically stable in $D$.
Lemma 3. For any $\left(S_{0}, I_{0}, R_{0}\right) \in D$, solution $(S, I, R)$ of (2) satisfies the following: 
(i) If $\sigma^{2}>(\beta \mu) / \Lambda$, then

$$
\underset{t \rightarrow \infty}{\limsup } \frac{1}{t} \ln \left[I+R+\left(\frac{\Lambda}{\mu}-S\right)\right] \leq \frac{\beta^{2}-2 \mu \sigma^{2}}{2 \sigma^{2}} .
$$

(ii) If $\sigma^{2} \leq(\beta \mu) / \Lambda$, then

$$
\underset{t \rightarrow \infty}{\limsup } \frac{1}{t} \ln \left[I+R+\left(\frac{\Lambda}{\mu}-S\right)\right] \leq \frac{\beta \Lambda}{\mu}-\mu-\frac{\Lambda^{2} \sigma^{2}}{2 \mu^{2}}
$$

Proof. Obviously, $(S, I, R) \in D$ for $t \geq 0$. Define

$$
V_{3}(S, I, R)=\ln \left[I+R+\left(\frac{\Lambda}{\mu}-S\right)\right] \text {. }
$$

Then,

$$
\begin{aligned}
\mathrm{LV}_{3} & =\frac{-\Lambda+2 \beta S f(I)+\mu S-\gamma_{1} I-\delta R-\left(\mu+\alpha+\gamma_{1}+\gamma_{2}\right) I-(\delta+\mu) R+\gamma_{2} I}{I+R+((\Lambda / \mu)-S)}-2\left[\frac{\sigma S f(I)}{I+R+((\Lambda / \mu)-S)}\right]^{2} \\
& =\frac{2 \beta S f(I)}{I+R+((\Lambda / \mu)-S)}+\frac{-\gamma_{1} I-\delta R-\left(\gamma_{1}+\alpha\right) I-\delta R}{I+R+((\Lambda / \mu)-S)}-\mu-2 \sigma^{2}\left[\frac{S f(I)}{R+I+((\Lambda / \mu)-S)}\right]^{2} \\
& \leq-\mu-2 \sigma^{2}\left[\frac{S f(I)}{R+I+((\Lambda / \mu)-S)}\right]^{2}+\frac{2 \beta S f(I)}{I+R+((\Lambda / \mu)-S)}
\end{aligned}
$$

Let $Z=(S f(I)) /(I+R+(\Lambda /(\mu-S)))$ and $\Psi(Z)=$ $-2 \sigma^{2} Z^{2}+2 \beta Z-\mu$. From $S+I+R \leq \Lambda / \mu$ and (3),

$$
Z \leq \frac{S I}{I+R+((\Lambda / \mu)-S)} \leq \frac{S I}{2(I+R)} \leq \frac{S}{2} \leq \frac{\Lambda}{2 \mu} .
$$

Let $r_{0}=\sup _{Z \in(0, \Lambda /(2 \mu))} \Psi(Z)$. Then,

$$
\begin{aligned}
\mathrm{d} V_{3}= & \mathrm{LV}_{3} \mathrm{~d} t+\frac{2 \sigma S f(I)}{I+R+((\Lambda / \mu)-S)} \mathrm{d} B(t) \leq \Psi(Z) \mathrm{d} t \\
& +2 \sigma Z \mathrm{~d} B(t) \leq r_{0} \mathrm{~d} t+2 \sigma Z \mathrm{~d} B(t),
\end{aligned}
$$

which yields

$$
\begin{aligned}
& \ln \left[I(t)+R(t)+\left(\frac{\Lambda}{\mu}-S(t)\right)\right] \leq \ln \left[I_{0}+R_{0}+\left(\frac{\Lambda}{\mu}-S_{0}\right)\right] \\
& \quad+r_{0} t+\int_{0}^{t} 2 \sigma Z(s) \mathrm{d} B(s) .
\end{aligned}
$$

From the strong law of large numbers,

$$
\lim _{t \rightarrow \infty} \frac{1}{t} \int_{0}^{t} 2 \sigma Z(s) \mathrm{d} B(s)=0 \text { a.s. }
$$

Then,

$$
\limsup _{t \rightarrow \infty} \frac{1}{t} \ln \left[R(t)+I(t)+\left(\frac{\Lambda}{\mu}-S(t)\right)\right] \leq r_{0} .
$$

Obviously, if $\sigma^{2}>(\beta \mu) / \Lambda$, then $r_{0}=\Psi\left(\beta /\left(2 \sigma^{2}\right)\right)=$ $\left(\beta^{2}-2 \mu \sigma^{2}\right) /\left(2 \sigma^{2}\right)$; if $\sigma^{2} \leq(\beta \mu) / \Lambda$, then $r_{0}=\Psi(\Lambda /(2 \mu))=$ $(\beta \Lambda) / \mu-\mu-\left(\Lambda^{2} \sigma^{2}\right) /\left(2 \mu^{2}\right)$. Lemma 3 holds.

By Lemma 3, the following result holds.

Theorem 2. Assume that (i) $\sigma^{2}>\max \left\{(\beta \mu) / \Lambda, \beta^{2} /(2 \mu)\right\}$

or

(ii) $2 \beta(\mu / \Lambda)\left(1-\mu^{2} /(\beta \Lambda)\right)<\sigma^{2} \leq(\beta \mu) / \Lambda$.

Then, disease-free equilibrium $E_{0}$ of (2) is almost surely exponentially stable in $D$.

\section{Remark 1}

(i) If $\mathscr{R}_{0}<1$ and $\sigma^{2}=0$, then $\left(\sigma^{2} \Lambda^{2}\right) /\left(2 \mu^{2}\right)<\left(\mu+\gamma_{1}+\right.$ $\left.\gamma_{2}+\alpha\right)\left(1-\mathscr{R}_{0}\right)$ holds. From Theorem 1 , if $\mathscr{R}_{0}<1$, then disease-free equilibrium $E_{0}$ of (1) is asymptotically stable in $D$. Hence, Theorem 1 extends Theorem 2.1 in [15].

(ii) From Theorem 2, if $\mathscr{R}_{0}<1, \beta \Lambda<\mu^{2}$, then diseasefree equilibrium $E_{0}$ of (1) is exponentially stable in $D$. Hence, Theorem 2 partially improves Theorem 2.1 in [15].

(iii) From Theorem 1, if $\gamma_{1}>\left(\sigma^{2} \Lambda^{2}\right) /\left(2 \mu^{2}\right)+\beta(\Lambda / \mu)-$ $\mu-\gamma_{2}-\alpha$, then disease-free equilibrium $E_{0}$ of (2) is stochastically asymptotically stable in $D$.

\section{Remark 2}

(i) Assume that $((2 \beta \mu) / \Lambda)\left[1-\mu\left(\mu+\gamma_{1}+\gamma_{2}+\alpha\right) /\right.$ $(\Lambda \beta)]>\beta^{2} /(2 \mu)$ and $\mathscr{R}_{0}>2$. From condition $(i)$ in Theorem 2, if $\sigma^{2}>\max \left\{(\beta \mu) / \Lambda, \beta^{2} /(2 \mu)\right\}=\beta^{2} /(2 \mu)$, then disease-free equilibrium $E_{0}$ of (2) is almost surely exponentially stable in $D$. However, Theorem 2 in [26] implies that disease of (2) will become extinct if $\left(C_{3}\right)$ of Theorem 2 in [26] holds, i.e., $\sigma^{2}>\max \{((\beta \mu) /$ $\left.\Lambda) \cdot\left(\mathscr{R}_{0} / 2\right),((2 \beta \mu) / \Lambda)\left(1-\mu\left(\mu+\gamma_{1}+\gamma_{2}+\alpha\right) /(\Lambda \beta)\right)\right\}$. 
(ii) Assume that $\beta^{2} /(2 \mu)<(\beta \mu) / \Lambda<((2 \beta \mu) / \Lambda)(1-\mu$ $\left.\left(\gamma_{1}+\gamma_{2}+\alpha+\mu\right) /(\Lambda \beta)\right)$ and $\mathscr{R}_{0}<2$. From Theorem $2(i), E_{0}$ is almost surely exponentially stable in $D$ if $\sigma^{2}>\max \left\{(\beta \mu) / \Lambda, \beta^{2} /(2 \mu)\right\}=(\beta \mu) / \Lambda$, whereas disease will become extinct with probability one if $\sigma^{2}>\max \left\{(\beta \mu) / \Lambda,((2 \beta \mu) / \Lambda)\left(1-\mu\left(\mu+\gamma_{1}+\gamma_{2}+\alpha\right) /\right.\right.$ $(\Lambda \beta))\}$ in $[26]$.

Obviously, condition (i) of Theorem 2 is weaker than condition $\left(C_{3}\right)$ of Theorem 2 in [26].

Remark 3. Let $\beta^{2} /\left(2\left(\gamma_{1}+\gamma_{2}+\mu+\alpha\right)\right)>((2 \beta \mu) / \Lambda)\left(1-\mu^{2}\right.$ $/(\beta \Lambda))$. By Theorem $2, E_{0}$ is almost surely exponentially stable in $D$ if condition (ii) holds. However, disease will become extinct if $\max \left\{\beta^{2} /\left(2\left(\gamma_{1}+\gamma_{2}+\mu+\alpha\right)\right),((2 \beta \mu) / \Lambda)\right.$ $\left.\left(1-\mu\left(\gamma_{1}+\gamma_{2}+\mu+\alpha\right) /(\Lambda \beta)\right)\right\}<\sigma^{2}<(\beta \mu) / \Lambda$ in [26]. Thus, condition (ii) of Theorem 2 is weaker than condition $\left(C_{2}\right)$ of Theorem 2 in [26].

Remark 4. From Remarks 2 and 3, Theorem 2 partially improves Theorem 2 in [26].

\section{Asymptotic Properties around Endemic Equilibrium}

In studying epidemic dynamics, we have interest in persistence of epidemic. We consider the behavior of solutions to (2) around endemic equilibrium $E^{*}\left(S^{*}, I^{*}, R^{*}\right)$ of corresponding deterministic model (1). Denote

$$
\begin{aligned}
& a_{1}=\frac{\alpha}{\gamma_{2}}, \\
& a_{2}=\frac{\left[2 \mu\left(2 \mu+\gamma_{2}+\alpha\right)+2 \mu \delta+\alpha \delta\right] I^{*}}{\beta \delta f\left(I^{*}\right)}, \\
& a_{3}=\frac{2 \mu}{\delta} .
\end{aligned}
$$

Theorem 3. If $\mathscr{R}_{0}>1$ and $\sigma^{2}<\mu\left(a_{3}+1\right) /\left(a_{2} I^{*}\right)$, then

$$
\begin{aligned}
& \limsup _{t \rightarrow \infty} \frac{1}{t} \mathbb{E} \int_{0}^{t}\left[\eta_{1}\left(S-S^{*}\right)^{2}+\eta_{2}\left(I-I^{*}\right)^{2}+\eta_{3}\left(R-R^{*}\right)^{2}\right] \mathrm{d} s \\
& \quad \leq a_{2} I^{*}\left(S^{*}\right)^{2} \sigma^{2},
\end{aligned}
$$

where $(S, I, R)$ be the solution of (2) with $\left(S_{0}, I_{0}, R_{0}\right) \in \mathbb{R}_{+}^{3}$ and

$$
\begin{aligned}
& \eta_{1}=\left(a_{3}+1\right) \mu-a_{2} I^{*} \sigma^{2}, \\
& \eta_{2}=a_{3}\left(\mu+\gamma_{2}+\alpha\right)+\mu+\alpha, \\
& \eta_{3}=a_{1}(\mu+\delta)+\mu .
\end{aligned}
$$

$$
V_{4}(S, I, R)=a_{1} W_{1}(R)+a_{2} W_{2}(I)+a_{3} W_{3}(S, I)+W_{4}(S, I, R),
$$

where

$$
\begin{aligned}
W_{1}(R) & =\frac{1}{2}\left(R-R^{*}\right)^{2}, \\
W_{2}(I) & =I-I^{*}-I^{*} \ln \left(\frac{I}{I^{*}}\right), \\
W_{3}(S, I) & =\frac{1}{2}\left(S-S^{*}+I-I^{*}\right)^{2}, \\
W_{4}(S, I, R) & =\frac{1}{2}\left(S-S^{*}+R-R^{*}+I-I^{*}\right)^{2} .
\end{aligned}
$$

From It $\hat{o}$ formula, (3), and $\left(\mathrm{H}_{2}\right)$,

$$
\begin{aligned}
\mathrm{LW}_{1} & =\left(R-R^{*}\right)\left[\gamma_{2} I-(\mu+\delta) R\right] \\
& =-(\delta+\mu)\left(R-R^{*}\right)^{2}+\gamma_{2}\left(I-I^{*}\right)\left(R-R^{*}\right), \\
\mathrm{LW}_{2}= & \left(I-I^{*}\right)\left[-\left(\gamma_{1}+\gamma_{2} \mu+\alpha\right)+\beta S f(I) \frac{1}{I}\right]+\frac{1}{2} I^{*}\left(\sigma S f(I) \frac{1}{I}\right)^{2} \\
= & \left(I-I^{*}\right)\left[\beta\left(S-S^{*}\right) f\left(I^{*}\right) \frac{1}{I^{*}}\right. \\
& \left.+\beta S\left(f(I) \frac{1}{I}-f\left(I^{*}\right) \frac{1}{I^{*}}\right)\right]+\frac{1}{2} I^{*} \sigma^{2} S^{2}\left(f(I) \frac{1}{I}\right)^{2} \\
\leq & \beta\left(I-I^{*}\right)\left(S-S^{*}\right) f\left(I^{*}\right) \frac{1}{I^{*}} \\
& +\beta S\left(I-I^{*}\right)\left(f(I) \frac{1}{I}-f\left(I^{*}\right) \frac{1}{I^{*}}\right)+\sigma^{2} I^{*}\left(S-S^{*}\right)^{2} \\
& +\sigma^{2} I^{*}\left(S^{*}\right)^{2} \leq \beta\left(I-I^{*}\right)\left(S-S^{*}\right) f\left(I^{*}\right) \frac{1}{I^{*}} \\
& +\sigma^{2} I^{*}\left(S-S^{*}\right)^{2}+\sigma^{2} I^{*}\left(S^{*}\right)^{2},
\end{aligned}
$$$$
\mathrm{LW}_{3}=\left(S-S^{*}+I-I^{*}\right)\left[\Lambda+\delta R-\mu S-\left(\gamma_{2}+\mu+\alpha\right) I\right]
$$$$
=\left(S-S^{*}+I-I^{*}\right)\left[-\mu\left(S-S^{*}\right)\right.
$$$$
\left.+\delta\left(R-R^{*}\right)-\left(\gamma_{2}+\mu+\alpha\right)\left(I-I^{*}\right)\right]
$$$$
=-\mu\left(S-S^{*}\right)^{2}-\left(\gamma_{2}+\alpha+2 \mu\right)\left(S-S^{*}\right)\left(I-I^{*}\right)
$$$$
-\left(\gamma_{2}+\alpha+\mu\right)\left(I-I^{*}\right)^{2}+\delta\left(R-R^{*}\right)\left[\left(S-S^{*}\right)+\left(I-I^{*}\right)\right] \text {, }
$$

$$
\begin{aligned}
\mathrm{LW}_{4}= & {\left[R+I+S-\left(R^{*}+I^{*}+S^{*}\right)\right][\Lambda-\mu S-(\mu+\alpha) I-\mu R] } \\
= & \left(R-R^{*}+S-S^{*}+I-I^{*}\right)\left[-\mu\left(S-S^{*}\right)-\mu\left(R-R^{*}\right)\right. \\
& \left.-(\alpha+\mu)\left(I-I^{*}\right)\right] \\
= & -\mu\left(R-R^{*}\right)^{2}-\mu\left(S-S^{*}\right)^{2}-(\alpha+\mu)\left(I-I^{*}\right)^{2} \\
& -(\alpha+2 \mu)\left(I-I^{*}\right)\left(S-S^{*}\right)-2 \mu\left(R-R^{*}\right)\left(S-S^{*}\right) \\
& -(\alpha+2 \mu)\left(R-R^{*}\right)\left(I-I^{*}\right) .
\end{aligned}
$$

From (25)-(33), 


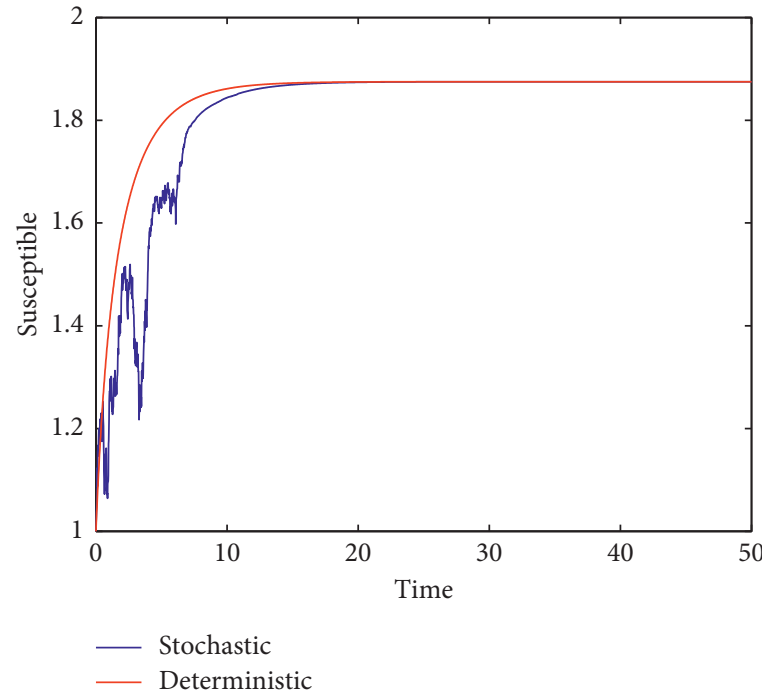

(a)

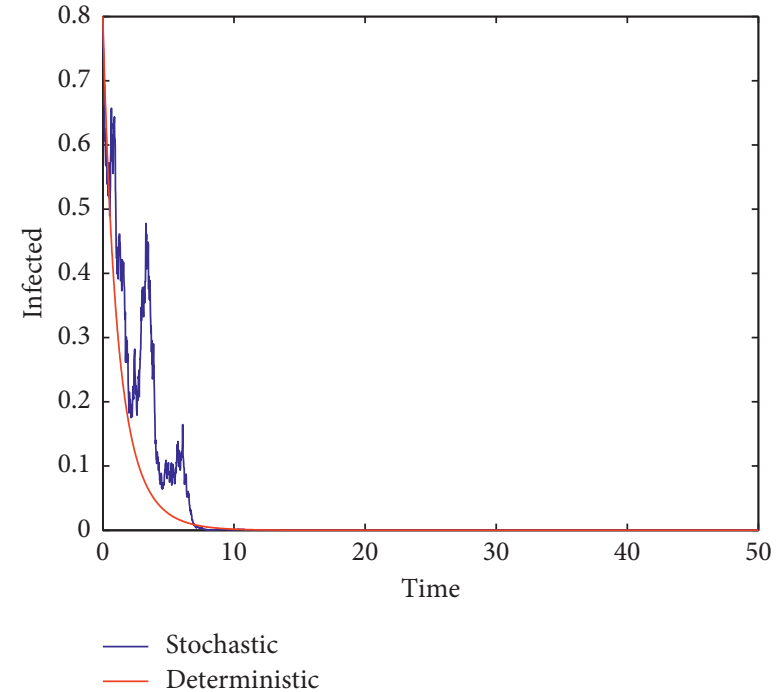

(b)

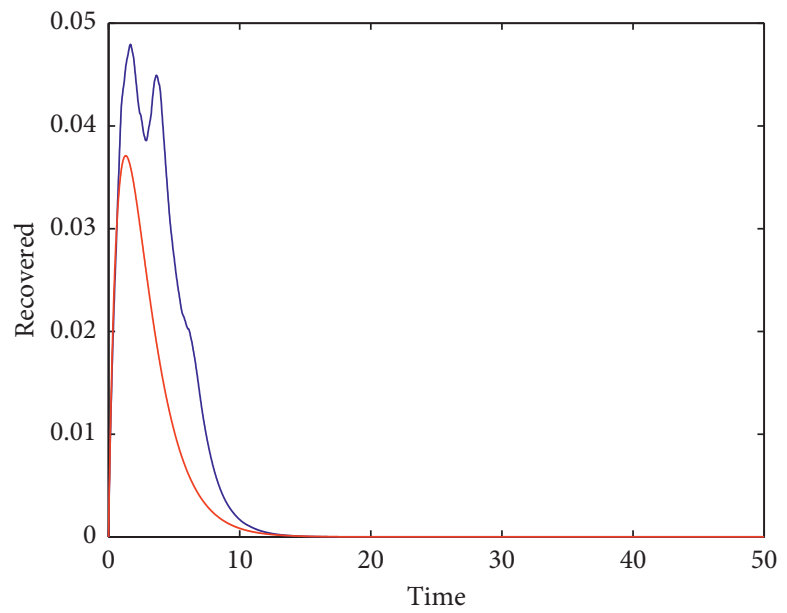

- Stochastic

Deterministic

(c)

FIgURE 1: Trajectories of deterministic model (1) and stochastic model (2) with (a) $\beta=0.3$, (b) $\gamma_{1}=0.57$, and (c) $\sigma^{2}=0.2$.

$$
\begin{aligned}
\mathrm{LV}_{4} \leq & a_{1} \gamma_{2}\left(R-R^{*}\right)\left(I-I^{*}\right)-a_{1}(\delta+\mu)\left(R-R^{*}\right)^{2}+\frac{a_{2} \beta f\left(I^{*}\right)}{I^{*}}\left(S-S^{*}\right)\left(I-I^{*}\right)+a_{2} I^{*} \sigma^{2}\left(S-S^{*}\right)^{2} \\
& +a_{2} I^{*} \sigma^{2}\left(S^{*}\right)^{2}-a_{3} \mu\left(S-S^{*}\right)^{2}-a_{3}\left(2 \mu+\alpha+\gamma_{2}\right)\left(I-I^{*}\right)\left(S-S^{*}\right)-a_{3}\left(\mu+\alpha+\gamma_{2}\right)\left(I-I^{*}\right)^{2} \\
& +a_{3} \delta\left(S-S^{*}\right)\left(R-R^{*}\right)+a_{3} \delta\left(R-R^{*}\right)\left(I-I^{*}\right)-\mu\left(S-S^{*}\right)^{2}-\mu\left(R-R^{*}\right)^{2} \\
& -(\alpha+\mu)\left(I-I^{*}\right)^{2}-(\alpha+2 \mu)\left(I-I^{*}\right)\left(S-S^{*}\right) \\
& -2 \mu\left(R-R^{*}\right)\left(S-S^{*}\right)-(\alpha+2 \mu)\left(R-R^{*}\right)\left(I-I^{*}\right) \\
= & -\left(a_{3} \mu+\mu-a_{2} I^{*} \sigma^{2}\right)\left(S-S^{*}\right)^{2}-\left[\mu+\alpha+a_{3}\left(\gamma_{2}+\alpha+\mu\right)\right]\left(I-I^{*}\right)^{2} \\
& -\left[\mu+a_{1}(\delta+\mu)\right]\left(R-R^{*}\right)^{2}+a_{2} I^{*}\left(S^{*}\right)^{2} \sigma^{2} \\
= & -\eta_{1}\left(S-S^{*}\right)^{2}-\eta_{2}\left(I-I^{*}\right)^{2}-\eta_{3}\left(R-R^{*}\right)^{2}+a_{2} I^{*}\left(S^{*}\right)^{2} \sigma^{2} .
\end{aligned}
$$




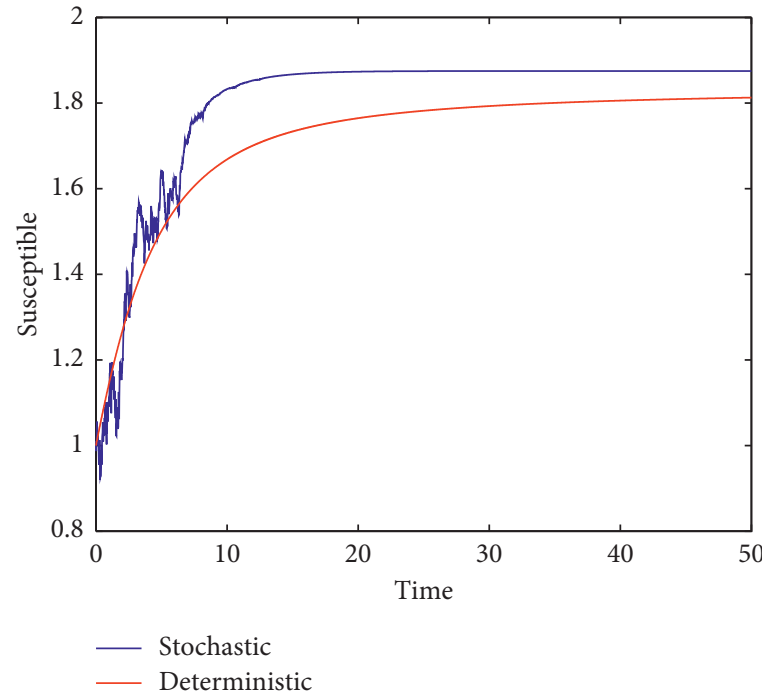

(a)

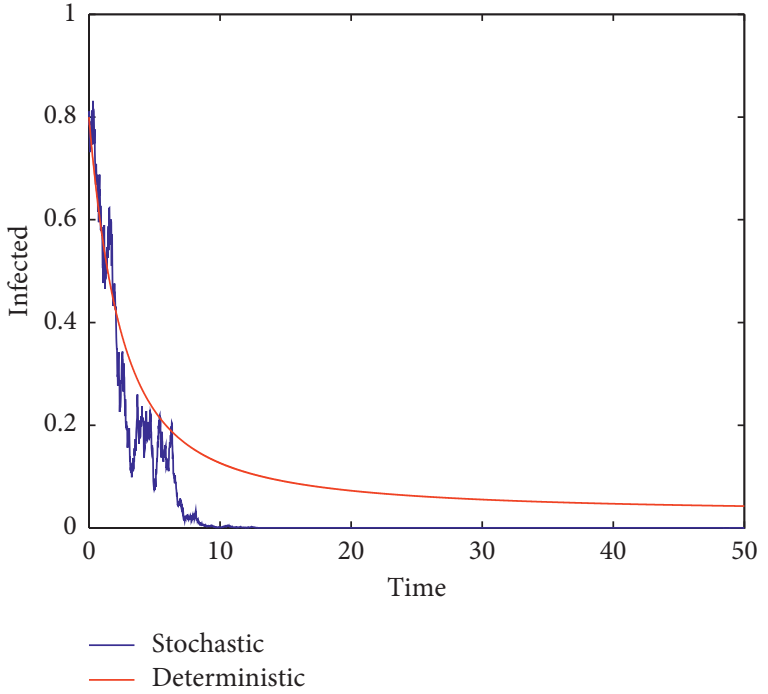

(b)

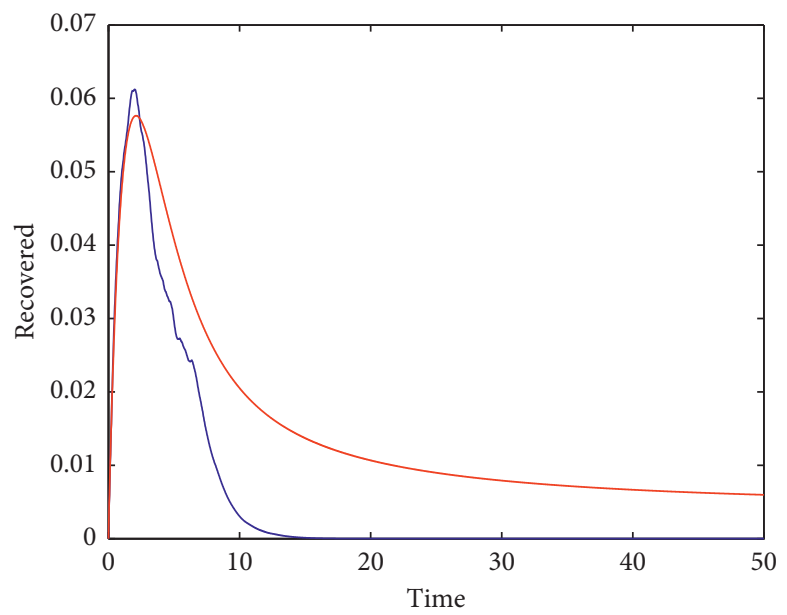

- Stochastic

— Deterministic

(c)

FIgURE 2: Trajectories of deterministic SIRS model (1) and stochastic SIRS model (2) with (a) $\beta=0.3$, (b) $\gamma_{1}=0.01$, and (c) $\sigma^{2}=0.3$.

Hence, we have

$$
\begin{gathered}
\mathrm{d} V(S, I, R) \leq\left[a_{2} I^{*}\left(S^{*}\right)^{2} \sigma^{2}-\eta_{1}\left(S-S^{*}\right)^{2}-\eta_{2}\left(I-I^{*}\right)^{2}\right. \\
\left.-\eta_{3}\left(R-R^{*}\right)^{2}\right] \mathrm{d} t+\sigma S\left(I-I^{*}\right) f(I) \frac{1}{I} \mathrm{~d} B(t) .
\end{gathered}
$$

It follows from (35) that

$$
\begin{aligned}
& V(S(t), I(t), R(t))-V\left(S_{0}, I_{0}, R_{0}\right) \\
& \leq \int_{0}^{t}\left[-\eta_{2}\left(I(s)-I^{*}\right)^{2}-\eta_{1}\left(S(s)-S^{*}\right)^{2}\right. \\
& \left.\quad-\eta_{3}\left(R(s)-R^{*}\right)^{2}\right] \mathrm{d} s \\
& \quad+a_{2} I^{*}\left(S^{*}\right)^{2} \sigma^{2} t+\int_{0}^{t} \sigma \frac{f(I(s))}{I(s)} S(s)\left(I(s)-I^{*}\right) \mathrm{d} B(s) .
\end{aligned}
$$

From (36),

$$
\begin{aligned}
\mathbb{E} V & (S(t), I(t), R(t))-\mathbb{E} V\left(S_{0}, I_{0}, R_{0}\right) \\
\leq & \mathbb{E} \int_{0}^{t}\left[-\eta_{2}\left(I(s)-I^{*}\right)^{2}-\eta_{1}\left(S(s)-S^{*}\right)^{2}\right. \\
& \left.-\eta_{3}\left(R(s)-R^{*}\right)^{2}\right] \mathrm{d} s+a_{2} I^{*}\left(S^{*}\right)^{2} \sigma^{2} t .
\end{aligned}
$$

Consequently,

$$
\begin{gathered}
\limsup _{t \rightarrow \infty} \frac{1}{t} \mathbb{E} \int_{0}^{t}\left[\eta_{1}\left(S(s)-S^{*}\right)^{2}+\eta_{2}\left(I(s)-I^{*}\right)^{2}\right. \\
\left.+\eta_{3}\left(R(s)-R^{*}\right)^{2}\right] \mathrm{d} s \leq a_{2} I^{*}\left(S^{*}\right)^{2} \sigma^{2} .
\end{gathered}
$$

Remark 5. Theorem 3 shows that if $\mathscr{R}_{0}>1, \sigma$ is small enough and then solution to (2) fluctuates around $E^{*}$; that is, disease will persist. Furthermore, if $\sigma=0$, then (34) becomes 


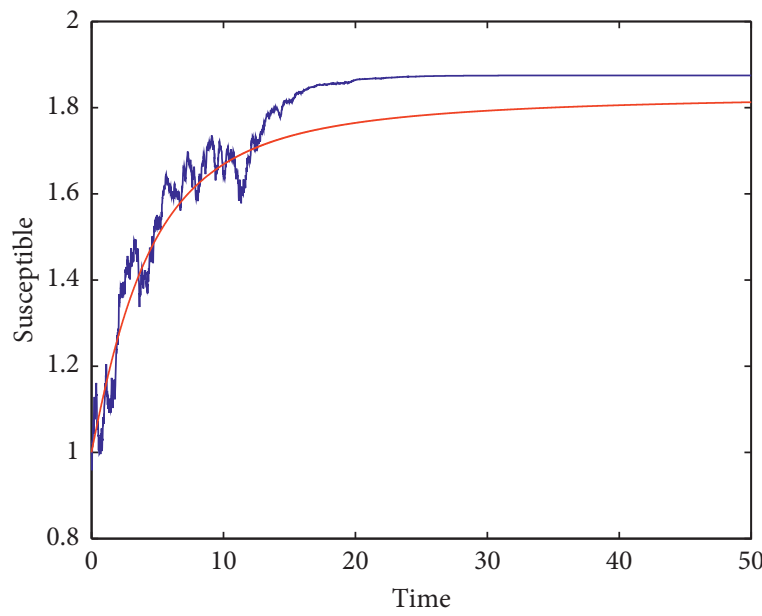

- Stochastic Deterministic

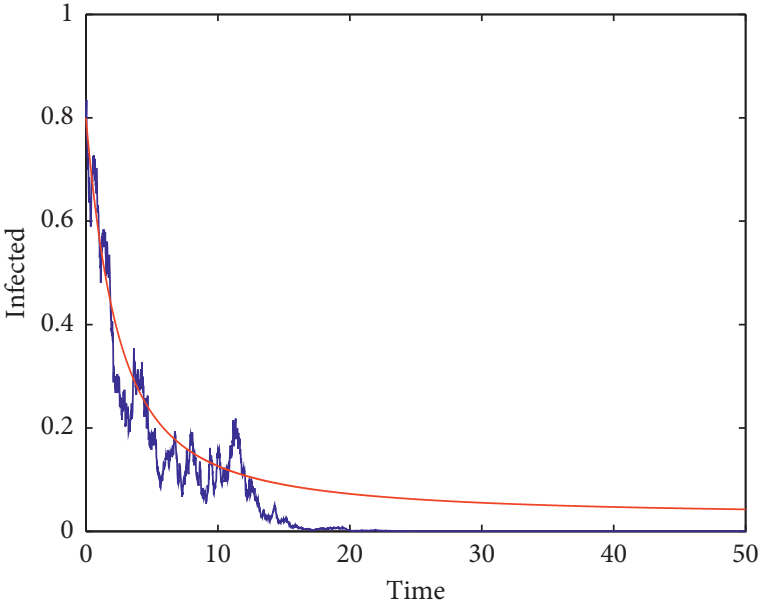

Stochastic

— Deterministic

(a)

(b)

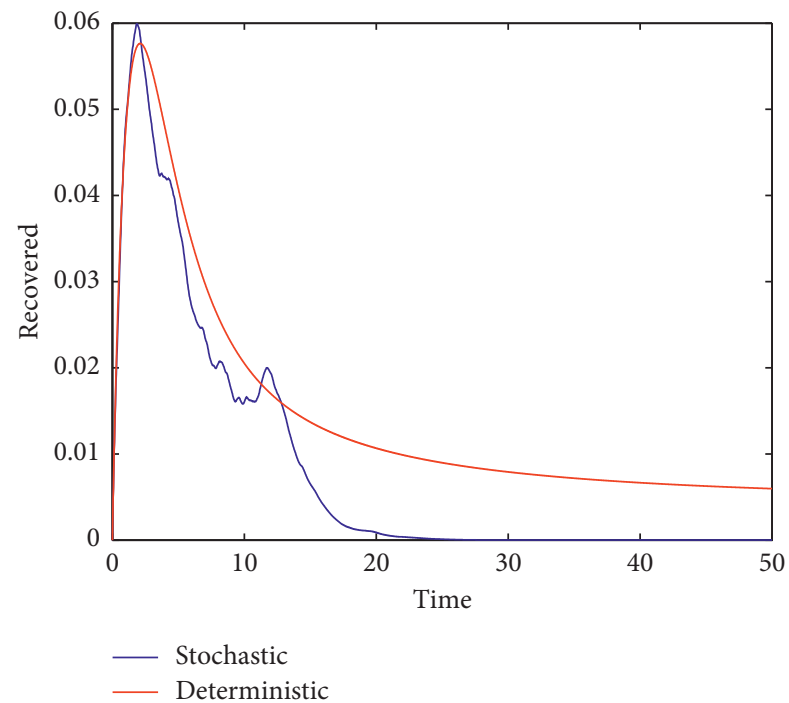

(c)

FIGURE 3: Trajectories of deterministic SIRS model (1) and stochastic SIRS model (2) with (a) $\beta=0.3$, (b) $\gamma_{1}=0.01$, and (c) $\sigma^{2}=0.15$.

$$
\mathrm{LV}_{4} \leq-\eta_{1}\left(S-S^{*}\right)^{2}-\eta_{2}\left(I-I^{*}\right)^{2}-\eta_{3}\left(R-R^{*}\right)^{2},
$$

which yields that for (1), $E^{*}$ is globally asymptotically stable in $\mathbb{R}_{+}^{3}$. This is consistent with Corollary 2.3 in [15]. Hence, Theorem 3 generalizes Corollary 2.3 in [15].

\section{Numerical Simulations}

By numerical simulation, we analyze the asymptotic behavior of model (2) so that readers can better understand our results. Let $f(I)=I /(1+I)$. Then, $I \geq f(I)$ for $I \geq 0$. Let

$$
\Lambda=0.6, \mu=0.32, \gamma_{2}=0.1, \alpha=0.1, \delta=0.4,
$$

$$
\left(S_{0}, I_{0}, R_{0}\right)=(1,0.8,0) \text {. }
$$

Example 1. Take $\beta=0.3, \gamma_{1}=0.57$, and $\sigma^{2}=0.2$. By a simple computation, we obtain $\mathscr{R}_{0} \approx 0.516<1$, $0.352 \approx\left(\sigma^{2} \Lambda^{2}\right) /\left(2 \mu^{2}\right)<\left(\mu+\gamma_{1}+\gamma_{2}+\alpha\right)\left(1-\mathscr{R}_{0}\right) \approx 0.5276$, $\beta \Lambda-\gamma_{1} \mu=-0.0024<0$, and $\gamma_{2}-\delta=-0.3<0$. Hence, the conditions of Theorem 1 hold. Furthermore, for (2), $E_{0}(1.875,0,0)$ is stochastically asymptotically stable. Figure 1 supports the result.

Example 2. Take $\beta=0.3, \gamma_{1}=0.01$, and $\sigma^{2}=0.3$. Hence, $\mathscr{R}_{0} \approx 1.0613>1$ and $\max \left\{(\beta \mu) / \Lambda, \beta^{2} /(2 \mu)\right\}=0.16<\sigma^{2}$. Then, according to conclusion (i) in Theorem 2 , solutions of (2) will tend almost surely exponentially to $E_{0}(1.875,0,0)$. However, from Corollary 2.3 in [15], the solution of deterministic model (1) will converge to $E^{*}(1.8261,0.0337,0.0047)$. This demonstrates that noises 


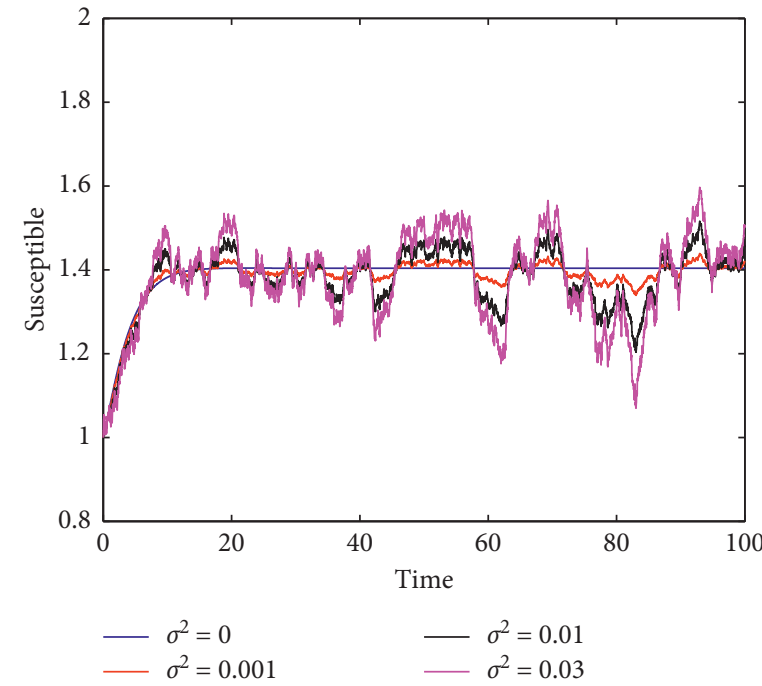

(a)

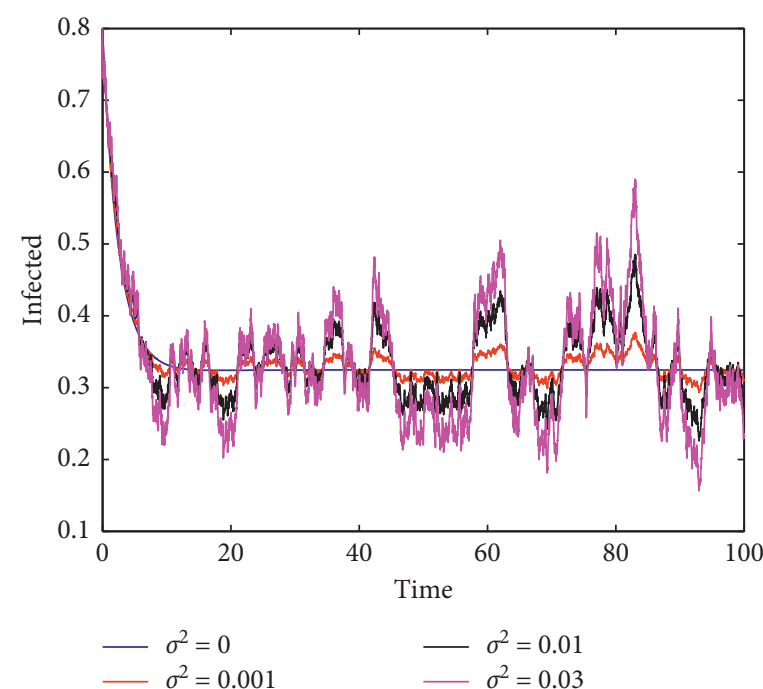

(b)

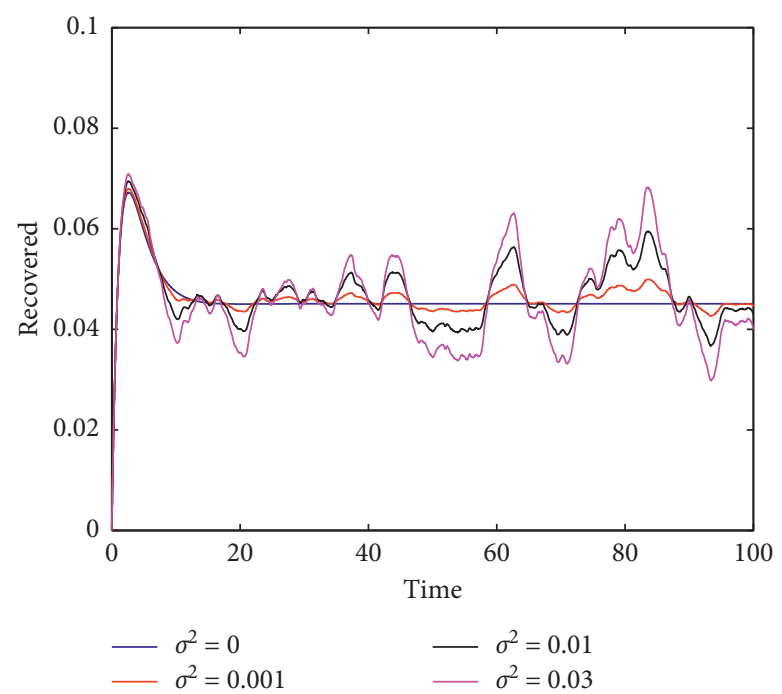

(c)

FIGURE 4: Trajectories of deterministic SIRS model (1) and stochastic SIRS model (2) for different $\sigma^{2}$ with $\beta=0.5$ and $\gamma_{1}=0.01$.

can result in extinction of disease. Figure 2 clearly supports these results.

Example 3. Let $\beta=0.3, \gamma_{1}=0.01$, and $\sigma^{2}=0.15$ such that $\mathscr{R}_{0} \approx 1.0613>1$ and $(2 \beta \mu / \Lambda)\left(1-\mu^{2} /(\beta \Lambda)\right) \approx 0.1380<\sigma^{2}<$ $(\beta \mu) / \Lambda=0.16$. Then, according to conclusion (ii) in Theorem 2 , solutions of (2) will tend almost surely exponentially to $E_{0}(1.875,0,0)$. However, from Corollary 2.3 in [15], endemic equilibrium $E^{*}(1.8261,0.0337,0.0047)$ of $(1)$ is globally asymptotically stable in $\mathbb{R}_{+}^{3}$. This represents the extinction of disease due to noise. Figure 3 clearly supports these results.

Example 4. Take $\beta=0.5, \gamma_{1}=0.01$. Then, $\mathscr{R}_{0} \approx 1.7689>1$ and $\sigma^{2}<\mu\left(a_{3}+1\right) /\left(a_{2} I^{*}\right) \approx 0.4644$. By Theorem 3, solutions of (2) fluctuate around endemic equilibrium $E^{*}(1.404$, $0.3245,0.0451)$ of deterministic model (1) in time average, which can be verified by using Figure 4 . In addition, Figure 4 shows that the fluctuation increases with increase in $\sigma^{2}$.

Example 5. Take $\gamma_{1}=0.01$. Figure 5 plots the average in time of infected $(1 / t) \int_{0}^{t} I(s) \mathrm{d} s$ for different $\beta$ in (a) and (b), respectively. From Figure 5 , the smaller the $\beta$ is, the smaller the number of infected cases is. In addition, when $\beta$ tends to 0 , the number of infected cases will tend to 0 . This result can also be derived from Theorem 2 .

Example 6. Take $\beta=0.5$. Figure 6 plots the average in time of infected $(1 / t) \int_{0}^{t} I(s) \mathrm{d} s$ for different $\gamma_{1}$ in (a) and (b), respectively. Figure 6 shows that the larger the $\gamma_{1}$ is, the 


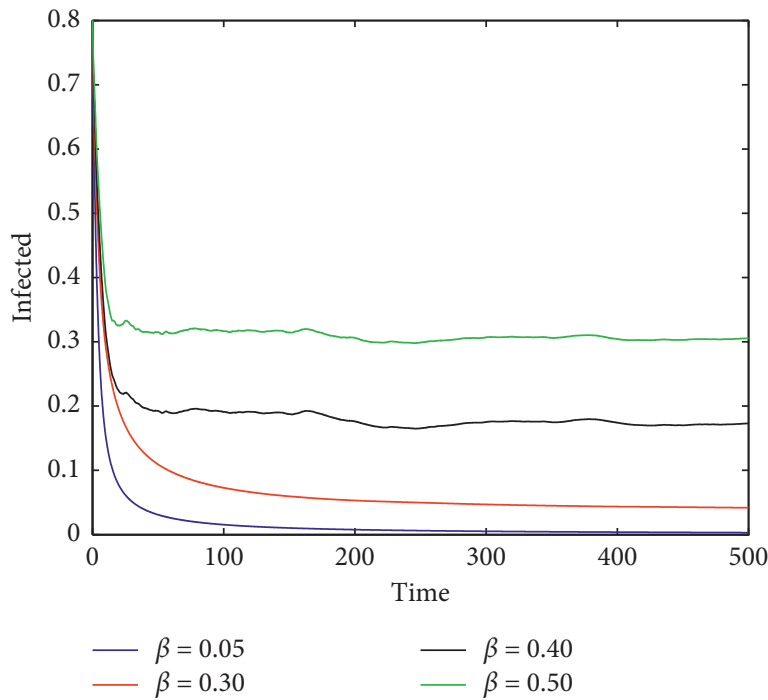

(a)

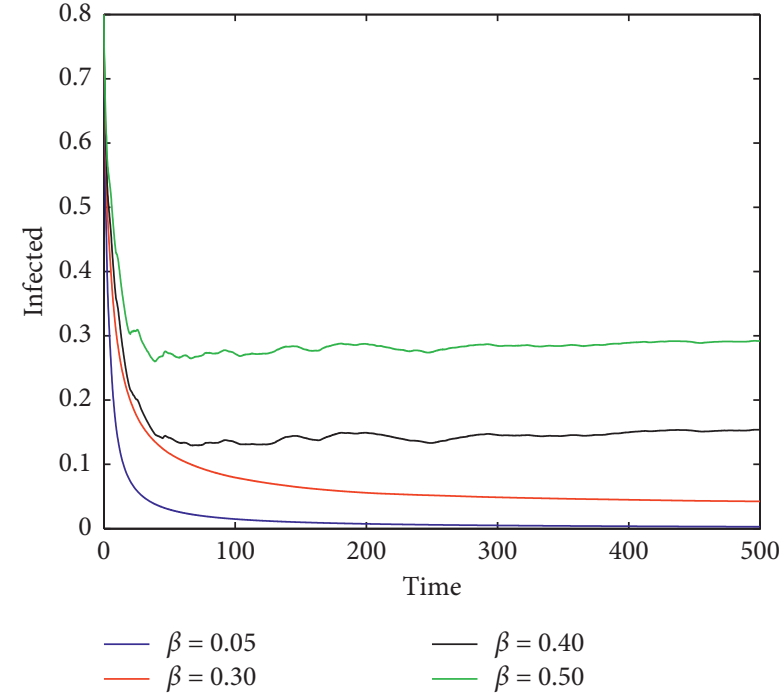

(b) FIGURE 5: Trajectories of the average in time of infected $(1 / t) \int_{0}^{t} I(s) \mathrm{d} s$ for stochastic SIRS model (2) for different $\beta$ with $\gamma_{1}=0.01$. (a)
$\sigma^{2}=0.02$. (b) $\sigma^{2}=0.04$.

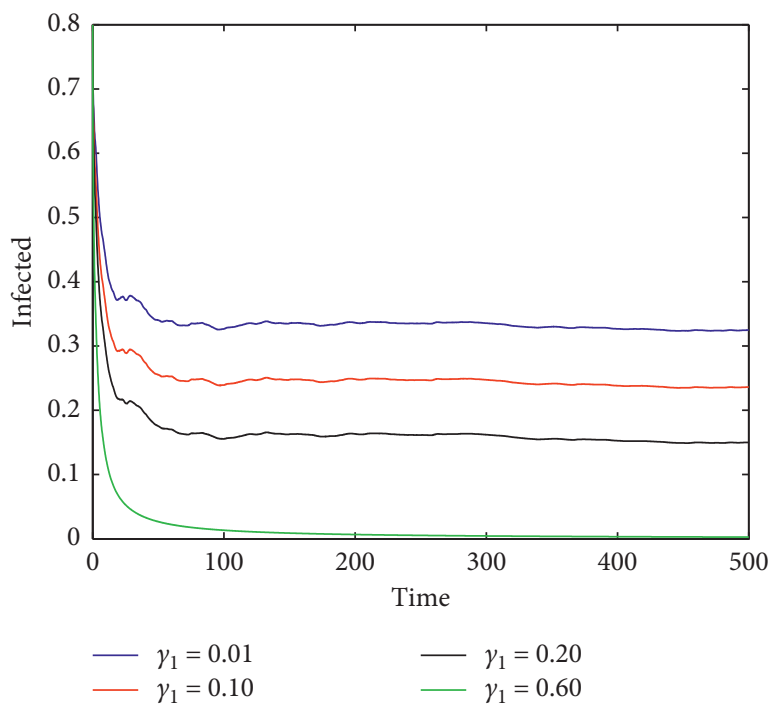

(a)

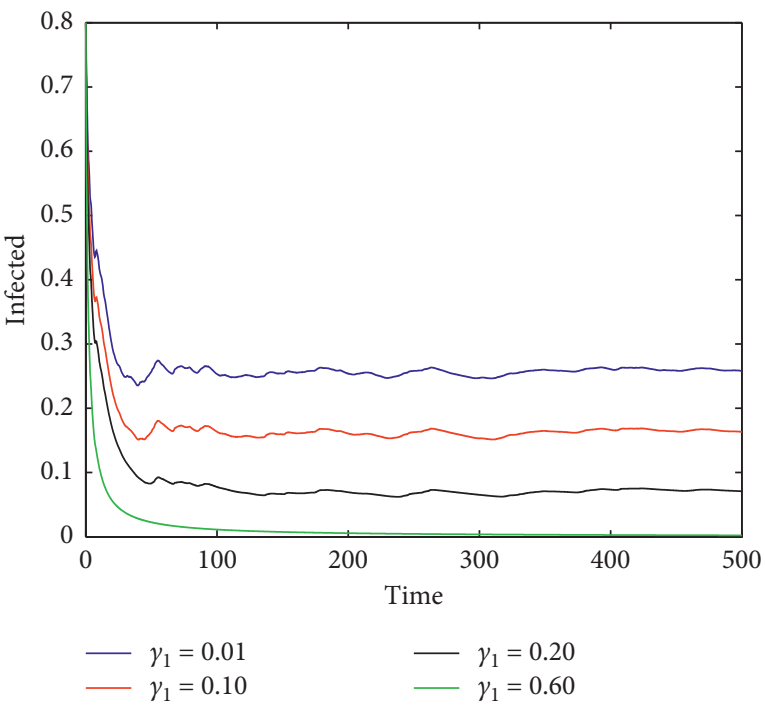

(b) FIGURE 6: Trajectories of the average in time of infected $(1 / t) \int_{0}^{t} I(s) \mathrm{d} s$ for stochastic SIRS model (2) for different $\gamma_{1}$ with $\beta=0.5$. (a)
$\sigma^{2}=0.02$. (b) $\sigma^{2}=0.1$.

smaller the number of infected cases is. Furthermore, when $\gamma_{1}$ is sufficiently large, the number of infected cases tends to 0 . This result can be derived from Remark 1 (iii).

Example 7. Take $\beta=0.3$. Figure 7 plots the number of infected cases for different $\sigma^{2}$ in (a) and (b), where $\gamma_{1}=$
0.01 and $\mathscr{R}_{0} \approx 1.0613>1$ in $\quad$ (a) and $\gamma_{1}=0.02$ and $\mathscr{R}_{0} \approx 1.0417>1$ in (b). From Corollary 2.3 in [15], endemic equilibrium of deterministic model (1) is globally asymptotically stable in $\mathbb{R}_{+}^{3}$. Figure 7 shows that $\sigma^{2}$ has a significant effect on both extinction and persistence of disease. 


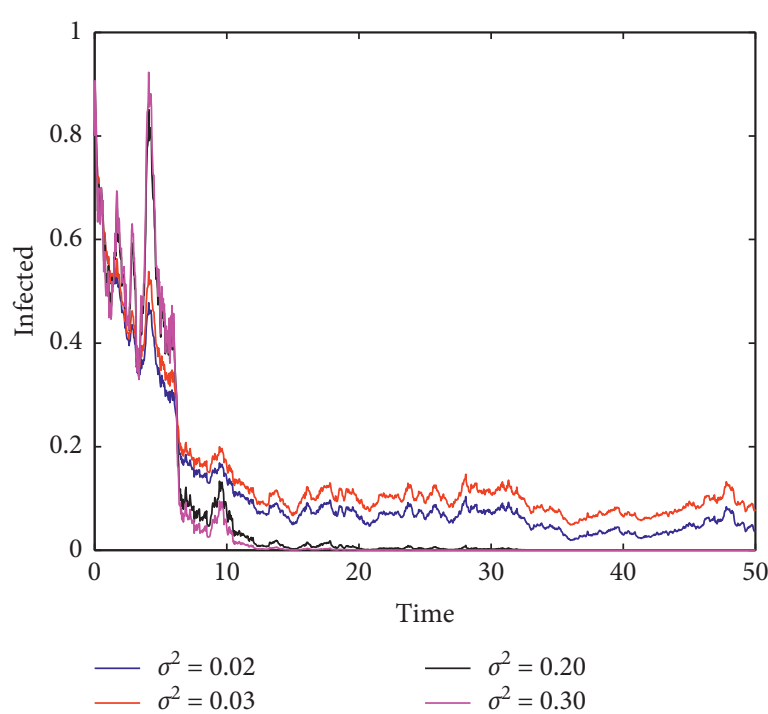

(a)

FIGURE 7: Trajectories of infected of stochastic SIRS model

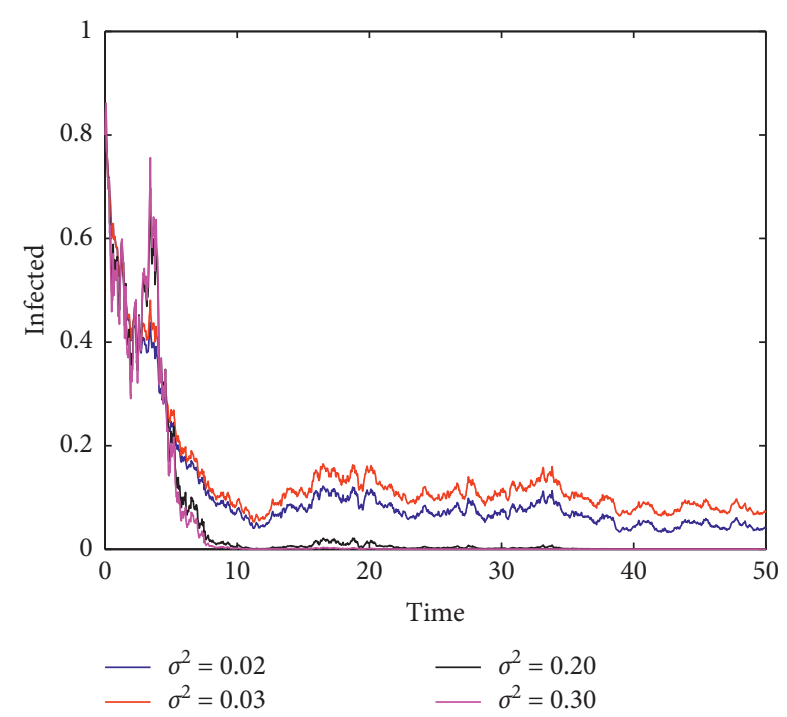

(b)

(2) for different $\sigma^{2}$ with $\beta=0.3$. (a) $\gamma_{1}=0.01$. (b) $\gamma_{1}=0.02$.

\section{Conclusions}

Stability is one of the important topics encountered in applications. However, because of the complexity of stochastic dynamics, there are not many results on stability analysis of stochastic differential equations.

Based on this, we investigate stochastic stability of a stochastic SIRS model. To begin with, using stochastic stability theory, we study stochastic asymptotic stability of disease-free equilibrium of (2), which generalizes Theorem 2.1 in [15]. Moreover, if the transfer rate from infectious to susceptible is sufficiently large, disease goes extinct. Then, exponential stability of disease-free equilibrium is obtained. This result partially improves Theorem 2.1 in [15] and Theorem 2 in [26] and demonstrates that noises can result in extinction of the disease. Furthermore, by the Lyapunov method, we give conditions to ensure that solution of (2) fluctuates around endemic equilibrium of (1) in time average. This generalizes Corollary 2.3 in [15]. At last, numerical simulations are presented to confirm theoretical results and find new properties.

Figure 4 shows that if $\mathscr{R}_{0}>1$ and $\sigma^{2}<\mu\left(a_{3}+1\right) /\left(a_{2} I^{*}\right)$, then the solution of (2) fluctuates around endemic equilibrium of (1). Moreover, Figure 4 also shows that the fluctuation increases with increase in noise intensity. From Figure 5, the smaller the $\beta$ is, the smaller the number of infected individuals will be. In addition, when $\beta$ tends to 0 , the number of infected individuals will tend to 0 . This result can also be derived from Theorem 2. Figure 6 shows that the larger the $\gamma_{1}$ is, the smaller the number of infected will be. Furthermore, when $\gamma_{1}$ is sufficiently large, the number of infected tends to 0 . This result can be derived from Remark 1 (iii). Figure 7 shows that noise intensity has a significant effect on both extinction and persistence of the disease. Hence, noises play a vital role in epidemic transmission.

For deterministic SIRS model (1), $\mathscr{R}_{0}$ is the basic reproduction number. However, for stochastic SIRS model (2), $\mathscr{R}_{0}$ is not a threshold parameter. From Theorem 2, no matter what the value of $\mathscr{R}_{0}$ is, the disease could go extinct. This can also be verified by the examples in this paper.

Although there are important findings revealed by the above investigation, the results still have some limitations. One may consider stochastic asymptotic stability in $\mathbb{R}_{+}^{3}$. In addition, our numerical simulation results show that the disease goes extinct as long as $\mathscr{R}_{0}<1$. Regrettably, our theoretical results do not lead to this conclusion.

\section{Data Availability}

No data were used to support this study.

\section{Conflicts of Interest}

The authors declare that they have no conflicts of interest.

\section{Authors' Contributions}

All the authors contributed equally and significantly in writing this paper. All authors read and approved the final manuscript.

\section{Acknowledgments}

This work was supported by the National Natural Science Foundation of China (No. 11971279). 


\section{References}

[1] R. Liu and G. Liu, "Asymptotic behavior of a stochastict two species competition model under the effect of disease," Complexity, vol. 2018, Article ID 3127404, 15 pages, 2018.

[2] Q. Zhang, X. Wen, D. Jiang, and Z. Liu, "The stability of a predator-prey system with linear mass-action functional response perturbed by white noise," Advances in Difference Equations, vol. 2016, 2016.

[3] R. Liu and G. Liu, "Analysis on stochastic food-web model with intraguild predation and mixed functional responses," Physica A, vol. 531, Article ID 121782, 2019.

[4] C. C. McCluskey, "Complete global stability for an SIR epidemic model with delay-distributed or discrete," Nonlinear Analysis: Real World Applications, vol. 11, no. 1, pp. 55-59, 2010.

[5] A. Lahrouz, L. Omari, D. Kiouach, and A. Belmaâti, "Complete global stability for an SIRS epidemic model with generalized non-linear incidence and vaccination," Applied Mathematics and Computation, vol. 218, no. 11, pp. 65196525, 2012.

[6] M. Y. Li, J. R. Graef, L. Wang, and J. Karsai, "Global dynamics of a SEIR model with varying total population size," Mathematical Biosciences, vol. 160, no. 2, pp. 191-213, 1999.

[7] Y. Muroya, Y. Enatsu, and Y. Nakata, "Global stability of a delayed SIRS epidemic model with a non-monotonic incidence rate," Journal of Mathematical Analysis and Applications, vol. 377, no. 1, pp. 1-14, 2011.

[8] W. Ma, M. Song, and Y. Takeuchi, "Global stability of an SIR epidemicmodel with time delay," Applied Mathematics Letters, vol. 17, no. 10, pp. 1141-1145, 2004.

[9] Windarto, M. A. Khan, M. Altaf Khan, and A. Fatmawati, "Parameter estimation and fractional derivatives of dengue transmission model," AIMS Mathematics, vol. 5, no. 3, pp. 2758-2779, 2020.

[10] M. A. Khan and A. Atangana, "Modeling the dynamics of novel coronavirus (2019-nCov) with fractional derivative," Alexandria Engineering Journal, vol. 34, 2020.

[11] J. Li, Z. Ma, and Z. Ma, "Stability analysis for SIS epidemic models with vaccination and constant population size," Discrete \& Continuous Dynamical Systems-B, vol. 4, no. 3, pp. 635-642, 2004.

[12] M. A. Khan, S. Ullah, S. Ullah, and M. Farhan, "Fractional order SEIR model with generalized incidence rate," AIMS Mathematics, vol. 5, pp. 2843-2857, 2020.

[13] A. Lahrouz, L. Omari, and D. Kiouach, "Global analysis of a deterministic and stochastic nonlinear SIRS epidemic model," Nonlinear Analysis: Modelling and Control, vol. 16, no. 1, pp. 59-76, 2011.

[14] Q. Liu and Q. Chen, "Analysis of the deterministic and stochastic SIRS epidemic models with nonlinear incidence," Physica A: Statistical Mechanics and its Applications, vol. 428, pp. 140-153, 2015.

[15] T. Li, F. Zhang, H. Liu, and Y. Chen, "Threshold dynamics of an SIRS model with nonlinear incidence rate and transfer from infectious to susceptible," Applied Mathematics Letters, vol. 70, pp. 52-57, 2017.

[16] Q. Liu, D. Jiang, N. Shi, T. Hayat, and A. Alsaedi, "Stationary distribution and extinction of a stochastic SIRS epidemic model with standard incidence," Physica A: Statistical Mechanics and its Applications, vol. 469, pp. 510-517, 2017.

[17] Z. Chang, X. Meng, and X. Lu, "Analysis of a novel stochastic SIRS epidemic model with two different saturated incidence rates," Physica A: Statistical Mechanics and its Applications, vol. 472, pp. 103-116, 2017.
[18] Y. Cai, Y. Kang, M. Banerjee, and W. Wang, "A stochastic SIRS epidemic model with infectious force under intervention strategies," Journal of Differential Equations, vol. 259, no. 12, pp. 7463-7502, 2015.

[19] H. Qi, L. Liu, and X. Meng, "Dynamics of a nonautonomous stochastic SIS epidemic model with double epidemic hypothesis," Complexity, vol. 2017, Article ID 4861391, 2017.

[20] Y. Lin and D. Jiang, "Threshold behavior in a stochastic SIS epidemic model with standard incidence," Journal of Dynamics and Differential Equations, vol. 26, no. 4, pp. 10791094, 2014.

[21] D. Jiang, J. Yu, C. Ji, and N. Shi, "Asymptotic behavior of global positive solution to a stochastic SIR model," Mathematical and Computer Modelling, vol. 54, no. 1-2, pp. 221-232, 2011.

[22] Y. Zhao and D. Jiang, "The threshold of a stochastic SIRS epidemic model with saturated incidence," Applied Mathematics Letters, vol. 34, pp. 90-93, 2014.

[23] A. Lahrouz and A. Settati, "Necessary and sufficient condition for extinction and persistence of SIRS system with random perturbation," Applied Mathematics and Computation, vol. 233, pp. 10-19, 2014.

[24] Q. Lu, "Stability of SIRS system with random perturbations," Physica A: Statistical Mechanics and its Applications, vol. 388, no. 18, pp. 3677-3686, 2009.

[25] M. EI Fatini, A. Lahrouz, R. Pettersson, A. Settati, and R. Taki, "Stochastic stability and instability of an epidemic model with relapse," Applied Mathematics and Computation, vol. 316, pp. 326-341, 2018.

[26] Y. Wang, G. Liu, and G. Liu, "Dynamics analysis of a stochastic SIRS epidemic model with nonlinear incidence rate and transfer from infectious to susceptible," Mathematical Biosciences and Engineering, vol. 16, no. 5, pp. 6047-6070, 2019.

[27] Q. Liu and Q. Chen, "Dynamics of a stochastic SIR epidemic model with saturated incidence," Applied Mathematics and Computation, vol. 282, pp. 155-166, 2016.

[28] X. Mao, Stochsatic Differential Equations and Applications, Horwood, Chichester, UK, 2007. 